\title{
Atividade antimicrobiana, antioxidante e teor de compostos fenólicos em casca e amêndoa de frutos de manga
}

\author{
Kettelin Aparecida Arbos ${ }^{2}$, Pamela Caroline Stevani ${ }^{3}$, Raquel de Fátima Castanha ${ }^{3}$
}

\section{RESUMO}

O processamento industrial de frutos de manga gera elevada quantidade de resíduos agroindustriais, representados pelas cascas e sementes (amêndoa), os quais, sem aplicação viável, acabam sendo descartados diretamente no meio ambiente. Esses resíduos são ricos em compostos bioativos, amplamente reconhecidos pelas suas propriedades promotoras da saúde e em aplicações tecnológicas. Os objetivos foram avaliar as atividades antimicrobiana e antioxidante e o teor de compostos fenólicos de dois extratos: um obtido da casca (FC) e outro da amêndoa (FA) de manga variedade 'Tommy Atkins'. O teor de compostos fenólicos totais variou de 3.123 a 6.644 mg de catequina/100 g. Os extratos FC e FA demonstraram relevante atividade antimicrobiana frente às cepas das bactérias Escherichia coli, Salmonella sp., Pseudomonas aeruginosa e Staphylococcus aureus, determinadas pelo método de difusão em disco. A ação antioxidante de FC e FA aumentou com o aumento das concentrações testadas, atingindo o valor máximo de 88\% (FC). Em todas as concentrações testadas os extratos FC apresentaram ação antioxidante significativamente superior às respectivas concentrações dos extratos FA. Esses resultados sugerem potencial aplicação dos resíduos de manga como fonte de compostos fenólicos, substâncias antimicrobianas e antioxidantes, podendo ser explorados pelas indústrias de alimentos.

Palavras-chave: bioativos, resíduo industrial, Mangifera indica L., DPPH.

\section{ABSTRACT}

\section{Antimicrobial and antioxidant activity and total phenolic content in mango peel and kernel}

The industrial processing of mangoes generates high amount of agroindustrial waste in the form of peel and kernel, which, without feasible application, is eventually discharged directly into the environment. These residues are rich in bioactive compounds, widely recognized for their health-promoting activity and technological applications. The aim of this study was to evaluate the antimicrobial activity and phenolic composition of peel (FC) and kernel (FA) extracts from mango of variety 'Tommy Atkins'. Total phenolic compounds of the samples ranged between 3123 and $6644 \mathrm{mg}$ of catequin/ 100g. The extracts FC and FA showed good antimicrobial activity against bacteria Escherichia coli, Salmonella sp., Pseudomonas aeruginosa and Staphylococcus aureus as determined by the disc diffusion method. The results demonstrated that the antioxidant action of FC and FA increases with increasing concentrations, reaching a maximum value of $88 \%$ (FC). In all concentrations tested, the FC extracts showed antioxidant activity significantly higher than the respective concentrations of FA. The results suggest potential practical applications of mango residues as sources of phenolic compounds, antimicrobial and antioxidant agents and should be exploited by food industry.

Key words: bioactive, industrial residue, Mangifera indica L., DPPH.

\footnotetext{
Recebido para publicação em 14/05/2012 e aprovado em 15/01/2013.

${ }^{1}$ Trabalho de pesquisa de Iniciação Científica.

${ }^{2}$ Nutricionista, Doutora. Departamento de Tecnologia de Alimentos, Universidade Federal da Paraíba, Rua Francisco Diomedes, 21, Cabo Branco, 58045-210, João Pessoa, Paraíba, Brasil. kettelin@crdr.ufpb.br

${ }^{3}$ Graduandas em Nutrição. Departamento de Tecnologia de Alimentos, Universidade Federal da Paraíba, Rua Francisco Diomedes, 21, Cabo Branco, 58045-210, João Pessoa, Paraíba, Brasil.
} 


\section{INTRODUÇÃO}

Os resíduos resultantes do processamento agroindustrial de fontes vegetais podem representar significativa fonte poluidora, pois sem uma aplicação viável muitas vezes são descartados diretamente no meio ambiente (Vieira et al., 2009). Entretanto, alguns estudos têm demonstrado aplicações sustentáveis para esses subprodutos, sendo empregados na produção de fertilizantes orgânicos, ração animal, produção de etanol, enzimas, óleos essenciais e aditivos (Ferrari et al., 2004; Kobori \& Jorge, 2005; Alexandrino, et al., 2007; Rodrigues et al., 2009; Tuttobene et al., 2009; Liu et al., 2010).

$\mathrm{O}$ processamento de frutas gera grande quantidade de resíduos ricos em compostos bioativos, amplamente reconhecidos pelas suas propriedades promotoras de saúde e aplicações tecnológicas, como antioxidantes e antimicrobianos, representando, portanto, potenciais fontes naturais dessas substâncias (Jorge \& Malacrida, 2008), superando muitas vezes o teor nas porções comestíveis das frutas (Soong \& Barlow, 2004; Shui \& Leong, 2006).

A manga (Mangifera indica L.) está entre as 10 culturas mais plantadas no mundo, com previsão de produção de mais de 28,8 milhões de toneladas em 2014 (FAO, 2010). No Brasil, em 2009 a produção de manga atingiu mais um milhão de toneladas, sendo destinada ao consumo interno, à exportação e industrialização (IBGE, 2009).

O processamento industrial da manga para elaboração de polpas, néctar, chutney, geleia, fruta desidratada, entre outros derivados, resulta num grande volume de resíduo, que, embora seja biodegradável, necessita de um tempo mínimo para ser decomposto (Couto Filho et al., 2007). Os resíduos do processamento da manga são resultantes da remoção das cascas (12 a $15 \%$ do peso da fruta) e sementes com as amêndoas em seu interior (15 a 25\%) (Mirghani et al., 2009).

Considerando o grande volume de resíduos gerados após o processamento industrial da manga e a demanda da indústria alimentícia por bioativos de fontes naturais, o presente trabalho propôs avaliar a ação antimicrobiana e antioxidante do resíduo de manga variedade 'Tommy Atkins' e determinar o teor de fenólicos totais.

\section{MATERIAL E MÉTODOS}

\section{Material}

Para obtenção dos resíduos de manga variedade ‘Tommy Atkins' foram adquiridos $10 \mathrm{~kg}$ de frutas em estágio comercial de maturação, sendo elas submetidas à remoção das cascas e sementes contendo as amêndoas. As cascas e sementes foram pesadas e submetidas à secagem a $60^{\circ} \mathrm{C}$ em desidratador marca Skymsem por $3 \mathrm{~h}$. Após esse período, as sementes foram abertas para remoção das amêndoas, que foram submetidas a novo ciclo de se- cagem $\left(60^{\circ} \mathrm{C} / 3 \mathrm{~h}\right)$. As cascas desidratadas foram trituradas em moinhos de faca da marca siemens ta-2, $800 w$ de potência, passadas em tamis de 80 mesh para obtenção de um pó uniforme denominado farinha da casca de manga (FC), a qual foi acondicionada em um frasco de polietileno escuro e mantido sob congelamento $\left(-18{ }^{\circ} \mathrm{C}\right)$ durante o desenvolvimento das análises. Para obtenção da farinha de amêndoa (FA) foram seguidos os mesmos procedimentos, utilizando apenas as amêndoas retiradas do interior das sementes.

\section{Preparo dos extratos}

Para o preparo dos extratos das farinhas (FC e FA) empregou-se o sistema solvente etanol:água (80:20 v/v) em agitação contínua em shaker (160 rpm/2 h) à temperatura ambiente, conforme descrito por Bloor (2001). Na sequência, os extratos FC e FA foram centrifugados a 5.000 xg por 15 min., filtrados a vácuo em papel-filtro quantitativo (8 ìm Quanty), para retirar o sobrenadante final, concentrados em evaporador rotativo, em pressão reduzida a $40{ }^{\circ} \mathrm{C}$ e liofilizados (Labconco, Freezone 4.5) para eliminação total do solvente.

\section{Determinação dos compostos fenólicos totais}

Na determinação do teor de compostos fenólicos totais, foi adotado procedimento proposto por Wettasinghe \& Shahidi (1999). Para a obtenção das curvas analíticas lineares, foi utilizada uma solução estoque de catequina na concentração de $1 \mathrm{mg} / \mathrm{mL}$. As soluções estoques foram diluídas de modo a obter concentrações de 0,1 até $1 \mathrm{mg}$ do equivalente de catequina/ $\mathrm{mL}$. Os teores de compostos fenólicos totais foram expressos em equivalentes de catequina/ 100 g de FC e FA, sendo as absorbâncias medidas a $773 \mathrm{~nm}$ em espectrofotômetro UV-visível marca FEMTO, modelo 800XI.

\section{Atividade antimicrobiana}

$\mathrm{Na}$ avaliação da atividade antimicrobiana, os extratos secos de cada fração (FC e FA) foram diluídos em água destilada estéril para a obtenção da concentração teste de $1 \mathrm{mg} / \mathrm{mL}$ e novamente esterilizados em filtro microbiológico com membrana Milipore $®$ de 0,22 ìm de porosidade. Para a realização da atividade antimicrobiana dos extratos de FC e FA foi utilizado o método de difusão em disco empregando quatro cepas bacterianas: Escherichia coli ATCC 25922, Salmonella sp. ATCC 14028, Pseudomonas aeruginosa ATCC 1014545 e Staphylococcus aureus ATCC 6538. As culturas de bactérias obtidas a partir de suspensões em caldo BHI com densidades ajustadas à turvação do tubo com 0,5 da escala de McFarland ( $\left.10^{8} \mathrm{UFC} / \mathrm{mL}\right)$ foram semeadas na superfície de placas de ágar Muller-Hinton (Difco), com auxílio de alça de drigalsky estéril, de modo a se obter cresci- 
mento uniforme e con-fluente. Em seguida, foram colocados sobre o meio discos de papel-filtro Whatman ${ }^{\circledR} \operatorname{com} 6$ mm de diâmetro, embebidos com $30 \mu \mathrm{L}$ dos extratos $(1 \mathrm{mg} /$ $\mathrm{mL}$ ), disco de controle positivo (antibiótico polimixina $300 \mu \mathrm{L}$ ) e disco de controle negativo (branco). A inibição do crescimento microbiano foi avaliada pelo diâmetro do halo de inibição ao redor dos discos após 24 horas de incubação a $37 \pm 1^{\circ} \mathrm{C}$. Os ensaios foram realizados em triplicatas, e os resultados foram expressos pela média aritmética dos valores dos halos obtidos nas três repetições.

\section{Atividade antioxidante}

A atividade antioxidante dos extratos foi mensurada de acordo com o método da redução do radical livre 1,1difenil-2-picrilhidrazil (DPPH), descrito por Koleva et al. (2002). Alíquotas de 2,5 mL das farinhas (FC e FA) nas concentrações de 0,25 a $2 \mathrm{mg} / \mathrm{mL}$ foram tratadas com 1 $\mathrm{mL}$ de solução etanólica de DPPH 0,3 mM. Após agitação, os tubos foram deixados em repouso ao abrigo da luz por 30 min. Decorrido o tempo de reação, a absorbância das amostras foi obtida em espectrofotômetro UV-visível marca FEMT, modelo 800XI, em comprimento de onda de 518 $\mathrm{nm}$ contra um branco específico para cada concentração (2,5 mL de extrato e 1,0 mL de etanol). A capacidade de sequestrar o radical livre foi expressa de duas formas: pelo valor da concentração inibitória $\left(\mathrm{IC}_{50}\right)$, que representa a quantidade de substância antioxidante necessária para reduzir em 50\% a concentração inicial de DPPH, e pelo percentual de atividade antioxidante (AA\%), conforme a equação 1 .

$\mathrm{AA} \%=100-\left\{\left[\left(\mathrm{Abs}_{\text {amostra }}-\mathrm{Abs}_{\text {branco }}\right) \times 100\right] / \mathrm{Abs}_{\text {controle }}\right\}$

\section{Análise estatística}

Os resultados foram expressos como média \pm desvio padrão. Para comparação das médias aritméticas, empregaram-se a análise de variância (ANOVA) e o teste Tukey, usando-se o software Prisma 4,0 (GraphPad). Adotou-se o nível de significância de $5 \%$ de probabilidade (p $<0,05)$.

\section{RESULTADOS E DISCUSSÃO}

\section{Compostos fenólicos totais nas cascas $e$ amêndoas de manga}

A quantidade de compostos fenólicos totais nos extratos dos resíduos de manga variou de 3.123 a 6.644 mg de catequina/ 100 g. O extrato obtido da FC apresentou teor de compostos fenólicos significativamente superior $(6.644,68 \pm 28,17 \mathrm{mg} / 100 \mathrm{~g})$ aos quantificados no extrato da FA $(3.123,13 \pm 30,21 \mathrm{mg} / 100 \mathrm{~g})$ e semelhante aos encontrados no extrato obtido do farelo da casca e da semente de manga $(5.800 \mathrm{mg} / 100 \mathrm{~g})$ quantificados por Vieira et al. (2009). O teor de fenólicos totais em farinhas obtidas de casca de manga (2.489 mg/ 100 g) (Araújo et al., 2009), acerola (279 mg/ $100 \mathrm{~g})$, graviola $(24,11 \mathrm{mg} / 100 \mathrm{~g})$ e abacaxi $(9,11 / 100 \mathrm{~g})$ (Souza \& Vieira, 2011) foi inferior ao dos extratos FC e FA.

Berardine et al. (2005) avaliaram o teor de compostos fenólicos de cascas de manga de 14 variedades e concluíram que as cascas da variedade 'Tommy Aktins' apresentaram o maior teor de polifenóis e pectina de alta qualidade. Esses autores sugeriram que as cascas devem ser rapidamente secas e submetidas à extração de compostos fenólicos para evitar degradações enzimática e microbiana. Adicionalmente, Souza \& Vieira (2011) sugeriram que resíduos de frutas devem ter seu uso implementado de forma a minimizar o desperdício de alimentos e gerar nova fonte alimentar.

\section{Atividade antimicrobiana}

A FC apresentou os maiores halos de inibição frente a todas as cepas avaliadas (gram-negativas ou positiva), superando a ação da FA, especialmente em relação às cepas de $P$. aeruginosa e $S$. aureus (Tabela 1). Semelhantemente, Khammuang \& Sarnthima (2011) demonstraram relevante ação antimicrobiana dos extratos dos resíduos de manga frente às cepas de $P$. aeruginosa .

Segundo Mothana \& Lindequist (2005), halos de inibição de 8 a 13 mm são considerados extratos com poder de ação moderadamente ativos, já halos de inibição entre $\geq 14 \mathrm{~mm}$ são extratos muito ativos. Com base nesse critério, os extratos FC e FA apresentaram elevada atividade antimicrobiana frente às bactérias avaliadas.

Muitos pesquisadores atribuem a ação antimicrobiana de extratos de origem vegetal ao teor de compostos fenólicos (Cabral et al., 2009; Al-Habib et al., 2010; Kumar et al., 2011; Martins, 2011).

Engels et al. (2009) e Mirghani et al. (2009) relataram o significante potencial antimicrobiano da amêndoa da manga, associando a ação antimicrobiana ao teor de taninos hidrolisados, os quais possuem capacidade de interação com proteínas, inibindo a atividade enzimática.

Dessa forma, é possível que a ação antimicrobiana dos extratos provenientes do resíduo agroindustrial da manga seja em parte atribuída ao seu alto teor de compostos fenólicos. Como já mencionado, o extrato FC apresentou maior capacidade de inibir o crescimento das cepas bacterianas testadas quando compradas à ação de FA, assim como mostrou maior teor de compostos fenólicos, corroborando os estudos de Kabuki et al. (2000), que associaram a relevante atividade antimicrobiana do resíduo de manga ao alto teor de polifenóis.

\section{Atividade antioxidante}

A ação antioxidante das farinhas (FC e FA) aumentou com o aumento das concentrações testadas, atin- 
gindo o valor máximo de $88,5 \pm 0,25 \%$ de atividade antioxidante para FC e de 69,8 $\pm 0,4 \%$ para FA, ambos na concentração de $2 \mathrm{mg} / \mathrm{mL}$. Em todas as concentrações testadas, FC apresentou ação antioxidante significativamente superior $(\mathrm{p} \leq 0,05)$ às respectivas concentrações de FA. Araújo et al. (2009) estudaram a ação antioxidante de extratos obtidos das cascas de três variedades de manga e verificaram redução do DPHH entre 50 e $80 \%$.

É possível que essa diferença deva-se ao elevado teor de compostos fenólicos encontrado na casca da manga (Ajila \& Prasada Rao, 2008; Berardine et al., 2005; Araújo et al., 2009), o que, em parte, justifica a ação antioxidante elevada do extrato FC; já o extrato FA possui alta concentração de substância lipídica com importante ação antimicrobiana já descrita.
Devido à variação no efeito antioxidante dos extratos da farinha de resíduo em razão das concentrações empregadas no ensaio, frequentemente os resultados são apresentados pelo valor de $\mathrm{IC}_{50}$, e quanto menor o valor de $\mathrm{IC}_{50}$ maior a atividade antioxidante do extrato. A correlação entre a atividade antioxidante (\%) e a concentração dos extratos de FC e FA resultou em um $\mathrm{IC}_{50}$ de $1,01 \mathrm{mg} /$ $\mathrm{mL}$ para $\mathrm{FC}\left(\mathrm{y}=79,111 \mathrm{x}, \mathrm{R}^{2}=0,912\right)$ e $\mathrm{IC}_{50}$ de $1,33 \mathrm{mg} / \mathrm{mL}$ $\left(y=36,58 x+0,985, R^{2}=0,9535\right)$ para FA (Figura 1). Comparando esses resultados com o valor de $\mathrm{IC}_{50}=8,0 \mathrm{mg} / \mathrm{mL}$ determinado em amostras de manga (Prado, 2009), podese verificar que as cascas e as amêndoas de manga avaliadas neste trabalho apresentaram atividade antioxidante superior às porções comestíveis, reforçando trabalhos anteriores (Soong \& Barlow, 2004; Ribeiro et al., 2007; Khammuang \& Sarnthima, 2011).

Tabela 1. Atividade antimicrobiana dos resíduos agroindustriais de frutos de manga

\begin{tabular}{|c|c|c|c|c|}
\hline \multirow[b]{2}{*}{ Extratos } & \multicolumn{4}{|c|}{ Diâmetro do halo de inibição $1 \mathrm{mg} / \mathrm{mL}(\mathrm{mm}) *$} \\
\hline & Escherichia coli & Salmonella sp. & $\begin{array}{c}\text { Staphylococcus } \\
\text { aureus }\end{array}$ & $\begin{array}{c}\text { Pseudomonas } \\
\text { aeruginosa }\end{array}$ \\
\hline FC & $17,1 \pm 0,15^{\mathrm{a}}$ & $18,1 \pm 0,15^{\mathrm{a}}$ & $19,0 \pm 0,1^{\mathrm{a}}$ & $19,1 \pm 0,15^{\mathrm{a}}$ \\
\hline FA & $15,5 \pm 0,02^{\mathrm{b}}$ & $16,1 \pm 0,11^{\mathrm{b}}$ & $14,9 \pm 0,2^{\mathrm{b}}$ & $14,2 \pm 0,1^{\mathrm{b}}$ \\
\hline
\end{tabular}

$\overline{\mathrm{FC}}=$ farinha da casca, $\mathrm{FA}$ = farinha do resíduo da amêndoa. Média de três determinações independentes. *Médias seguidas por letras iguais nas colunas não diferem estatisticamente $(\mathrm{p}<0,05)$

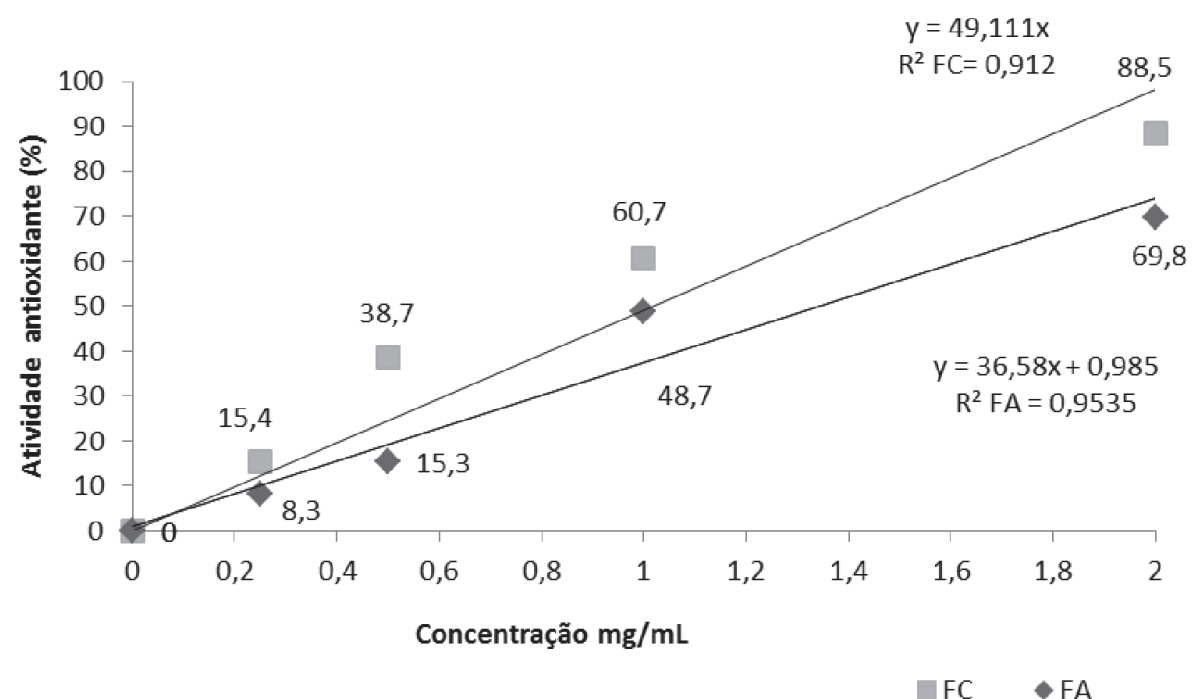

Figura 1. Avaliação da capacidade antioxidante da farinha de casca (FC) e farinha de amêndoa (FA) de frutos de manga pelo método de redução do radical DPPH.

\section{CONCLUSÃO}

Extratos provenientes dos resíduos do processamento industrial de frutos da manga (farinhas da casca e amêndoas) apresentaram teor importante de compostos fenólicos, atividade antioxidante e antimicrobiana.

Os extratos podem ser fonte potencial de bioativos, trazendo não apenas benefício nutricional como também redução do impacto ambiental devido ao aproveitamento desses resíduos para formulação de novos produtos pela indústria alimentícia.

\section{REFERÊNCIAS}

Ajila CM \& Prasada Rao UJ (2008) Protection against hydrogen peroxide induced oxidative damage in rat erythrocytes by Mangifera indica L. peel extract. Food and Chemical Toxicology, 46:303-309. 
Alexandrino AM, Faria HG, Souza CGM \& Peralta RM (2007) Aproveitamento do resíduo de laranja para a produção de enzimas lignocelulolíticas por Pleurotus ostreatus (Jack:Fr). Ciência e Tecnologia de Alimentos, 27:364-368.

Al-Habib A, Al-Saleh E, Safer A \& Afzal M (2010) Bactericidal effects of grape seed extracts on methicillin-resistant Staphylococcus aureus (MRSA). The Journal of Toxicological Sciences, 35:357-364.

Araújo CR, Melo EA, Lima GVLA \& Maciel MIS (2009) Resíduo agroindustrial de três variedades de mangas: teor de fitoquímicos e ação antioxidante. In: IX JEPEX - Jornada de ensino, pesquisa e extensão, Recife. Anais, UFRPE. p.42.

Berardine N, Fezer R, Conrad J, Beifuss U, Carle R \& Schieber A (2005) Screening of mango (Mangifera indica L.) cultivars for their contents of flavonol O- and xanthone C-glycosides, anthocyanins and pectin. Journal of Agricultural and Food Chemistry, 53: 1563-1570.

Bloor SJ (2001) Overview of methods for analysis and identification of flavonoids. Methods in Enzymology, 335:3-14.

Cabral ISR, Oldoni TLC, Prado A, Bezerra RMNB, Alencar SM, Ikegaki M \& Rosalen PL (2009) Composição fenólica, atividade antibacteriana e antioxidante da própolis vermelha brasileira. Química Nova, 32:1523-1527.

Couto Filho CCC, Silva Filho JC, Neiva Júnior AP, Freitas RTF, Souza RM \& Nunes JAR (2007) Qualidade da silagem de resíduo de manga com diferentes aditivos. Ciência e Agrotecnologia, 31:1537-1544.

Engels C, Knõdler M, Zhao YY, Carle R, Gänzle MG \& Schieber A (2009) Antimicrobial activity of gallotannins isolated from mango (Mangifera indica L.) kernels. Journal of Agricultural and Food Chemistry, 57:7712-7718.

FAO - Food and Agriculture Organization of the United Nations (2010) Current situation and medium-term outlook for tropical fruits. Disponível em: <http://www.fao.org/es/esc/common/ ecg/218/en/Sit_web_e.pdf >. Acessado em: 13 de novembro de 2012 .

Ferrari RA, Colussi F \& Ayub RA (2004) Caracterização de subprodutos da industrialização do maracujá-aproveitamento das sementes. Revista Brasileira de Fruticultura, 26:101-102.

IBGE - Instituto Brasileiro de Geografia e Estatística (2009) Áreas destinada à colheita e colhida, quantidade produzida, rendimento médio e valor da produção, segundo os principais produtos das lavouras permanentes Brasil - 2009. Disponível em: <http://www.ibge.gov.br/home/estatistica/economia/pam/2009/ tabelas_pdf/tabela03.pdf $>$. Acessado em: 13 de março de 2011.

Jorge N \& Malacrida CR (2008) Extratos de sementes de mamão (Carica papaya L.) como fonte de antioxidantes naturais. Alimentos e Nutrição, 19:337-340

Kabuki T, Nakajima H, Arai M, Ueda S, Kuwabara Y \& Shun D (2000) Characterization of novel antimicrobial compounds from mango (Mangifera indica L.) kernel seeds. Food Chemistry, 71:61-66

Khammuang S \& Sarnthima R (2011) Antioxidant and antibacterial activities of selected varieties of thai mango seed extract. Pakistan Journal of Pharmaceutical Sciences, 24:37-42.
Kobori CN \& Jorge N (2005) Caracterização dos óleos de algumas sementes de frutas como aproveitamento de resíduos industriais. Ciência e Agrotecnologia, 29:1008-1014.

Koleva LI, Van Beek TA, Linssen JP, Groot A \& Evstatieva LN (2002) Screening of plant extracts for antioxidant activity: a comparative study on three testing methods. Phytochemical Analysis, 13:8-17.

Kumar KA, Narayani M, Subanthini A \& Jayakumar M (2011) Antimicrobial activity and phytochemical analysis of citrus fruit peels - utilization of fruit waste. International Journal of Engineering Science and Technology, 3:5414-5421.

Liu K, Lin X, Yue J, Li X, Fang X, Lin J, Qu Y \& Xiao L (2010) High concentration ethanol production from corncob residues by fed-batch strategy. Bioresource Technology, 101:4952-4958.

Martins JGP (2011) Atividade antimicrobiana de produtos naturais: erva mate e resíduos agroindustriais. Dissertação de Mestrado. Universidade de São Paulo, São Paulo. 98p.

Mirghani MES, Yosuf F, Kabbashi NA, Vejayan J \& Yosuf ZBM (2009) Antibacterial activity of mango kernel extracts. Journal of Applied Sciences, 9:3013-3019.

Mothana RAA \& Lindequist U (2005) Antimicrobial activity of some medicinal plants of the island Soqotra. Journal of Ethnopharmacology Etnopharmacology, 96:177-181.

Prado A (2009) Composição fenólica e atividade antioxidante de frutas tropicais. Dissertação de Mestrado. Escola Superior de Agricultura "Luiz de Queiroz", Piracicaba. 106p.

Ribeiro SMR, Queiroz JH, Queiroz, MELR, Campos FM \& Sant'ana HMP (2007) Antioxidant in mango (Mangifera indica L.) pulp. Plant Foods for Human Nutrition, 62:13-17.

Rodrigues C, Vandenberghe LPS, Teodoro J, Oss JF, Pandey A \& Soccol CR (2009) A new alternative to produce gibberellic acid by solid state fermentation. Brazilian Archives of Biology and Technolology, 52:181-188.

Shui G \& Leong LP (2006) Residue from star fruit as valuable source for functional food ingredients and antioxidant nutraceuticals. Food Chemistry, 97:277-284.

Soong YY \& Barlow PJ (2004) Antioxidant activity and phenolic content of selected fruit seeds. Food Chemistry, 88:411-417.

Souza MSB \& Vieira LM (2011) Fenólicos totais e capacidade antioxidante in vitro de resíduos de polpas de frutas tropicais. Brazilian Journal of Food Technology, 14:202-210.

Tuttobene R, Avola G, Gresta F \& Abbate V (2009) Industrial orange waste as organic fertilizer in durum wheat. Agronomy for Sustainable Development, 29:557-563.

Vieira PAF, Queiroz JH, Vieira BC, Mendes FQ, Barbosa AA, Muller ES, Sant' ana RCO \& Moraes GHK (2009) Caracterização química do resíduo do processamento agroindustrial da manga (Mangifera indica L.) var. Ubá. Alimentos e Nutrição, 20:617623.

Wettasinghe M \& Shahidi F (1999) Evening primrose meal: a source of natural antioxidants and scavenger of hydrogen peroxide and oxygen-derived free radicals. Journal of Agricultural and Food Chemistry, 47:1801-1812. 